\title{
Preparation of Asymmetric Spiro Orthocarbonates via Cyclic Thionocarbonates
}

\author{
Chul-bae Kim, Min-Ah Park, Byungho Song, ${ }^{\dagger}$ and Kwangyong Park ${ }^{*}$ \\ Department of Chemical Engineering, Chung-Ang University, Seoul 156-756, Korea \\ "Department of Chemistr, Summoon University, Asan City, Ching Nam 336-708, Korea \\ Received May 28,2003
}

Key Words : Spiro orthocarbonate, SOC. Cyclic thionocarbonate, Asymmetric SOC

The development of monomers that show no shrinkage in volume upon polymerization is highly desirable in the field of materials such as dental fillings, high-strength composites. precision castings, and adhesives.' Volume shrinkage during polymerization results from the conversion of van der Waals distances between monomers to covalent bond lengths. Since the van der Waals distance separates monomer molecules approximately three times greater than a covalent bond does, the formation of new covalent bonds upon polymerization is destined to produce the substantial amount of volume contraction. A hopeful approach to prevent this shrinkage could be attained by using monomers that polymerize via the double ring-opening process, which results in two bonds breaking for each new bond formation.

Since the double ring-opening polymerization was reported in $1972 .^{2}$ various monomers based on spiro orthoester (SOE) ${ }^{3}$ bicyclo orthoester (BOE). ${ }^{4}$ polycyclic ketal lactone $(\mathrm{PKL}){ }^{5}{ }^{5}$ cyclic ether, ${ }^{6}$ spiroketal, ${ }^{7}$ and spiro orthocarbonate (SOC) skeletons ${ }^{8}$ have been developed to show the expansion in volume upon polymerization. Among them, spiro orthocarbonates (SOCs) are the most widely investigated monomers because they show the biggest volume expansion. Moreover SOCs containing exo-methylene groups undergo the ring-opening polymerization not only with cationic initiators ${ }^{\circ}$ but also with radical initiators. ${ }^{10}$ Asynmetric SOCs possessing one exo-methylene group are more favorable than symmetric SOCs, because the extra exomethylene group remained in the polymer from symmetric monomers affords the crosslinking reaction that causes the volume shrinkage. ${ }^{11}$ Recently, $N_{,} \mathrm{S}^{12}$ and $\mathrm{S}, \mathrm{S}$-analogs ${ }^{1.3}$ of SOCs have been introduced as alternatives.

A variety of synthetic approaches have been reported for the preparation of SOCS. The original syntheses of SOCS consist of organotin compounds. Reactions of the cyclic thionocarbonates with cyclic tin compounds produce asymmetric SOCs. ${ }^{14}$ Thallous alkoxide was also investigated for replacing oragnotins. ${ }^{15}$ New synthetic route for the preparation of cyclic thionocarbonates by using the reaction of thiophosgene with diols was developed by Stansbury, $e t$ al. ${ }^{\text {'' }}$ Another approach involves the transesterification utilizing tetramethyl or tetraethyl orthocarbonate, which supplies the spiro carbon, with two equivalents of diols in the presence of an acid catalyst. ${ }^{17}$ Endo developed a

"To whom all correspondences should be addressed. E-Inail: kypark $\overline{a c a u . a c . k r}$ modified route using dichlorodiphenoxymethane as a suitable way for the preparation of asymmetric SOCs. ${ }^{8 c, 10 c, 18}$ In spite of these numerous efforts, an efficient synthetic route for these monomers is still under investigation.

During a program directed toward the development of expandable monomers, we have explored various synthetic methods for the preparation of asymmetric SOCs bearing exo-methylene groups at $\alpha$ to the oxygen. The reaction of cyclic thionocarbonates with cyclic tin compounds gave the best results among those methods.

The preparation of cyclic thionocarbonate intermediates 2 was initially investigated. Even though it has been known that they could be prepared by the reaction of bis(tributyltin) alkylene glycolates with carbon disulfide, ${ }^{140}$ this approach did not give a good result in our case. The intermediates 2 could be prepared by the reaction of appropriate diols 1 with thiophosgene in the presence of 4-dimethylaminopyridine (DMAP) in good yields (Table 1).

Thionocarbonates 2a, 2b and $\mathbf{2 d}$ are produced in good yields (entry 1, 2 and 4). Pentagonal thionocarbonates 2a and $2 \mathbf{d}$ can be recrystallized from $10 \%$ hexane in ether, while hexagonal compound $\mathbf{2} \mathbf{b}$ is recrystallized from ether. The desired product $2 \mathrm{c}$ containing seven-membered ring was

Table 1. Preparation of Cyclic Thionocarbonates 2

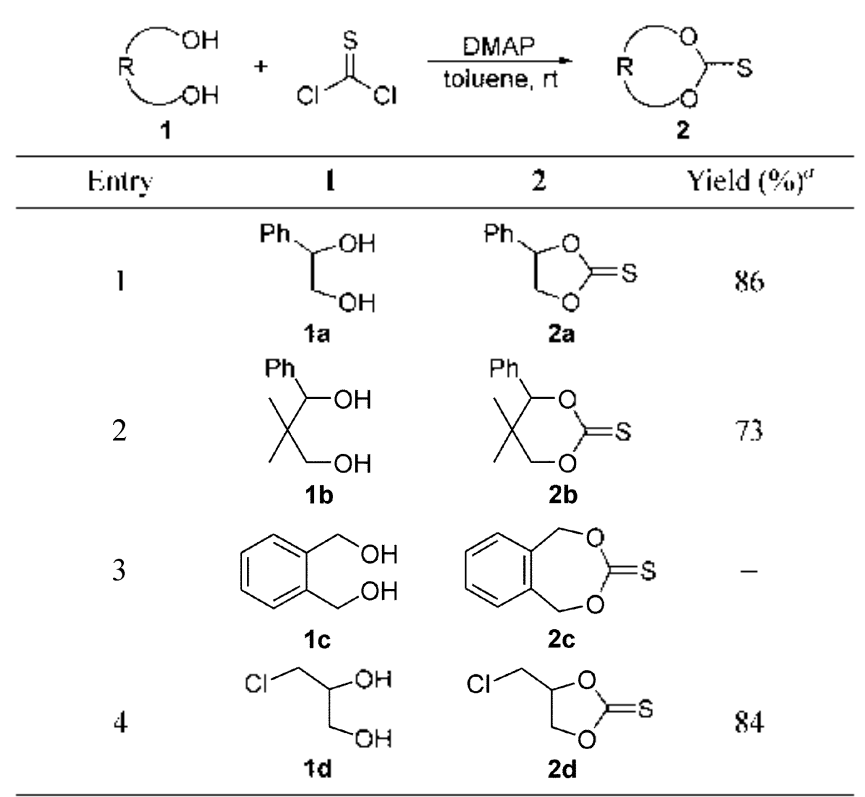

"The $y$ ields reler to isolated pure materials and based on thiophosgene. 
Table 2. Preparation of Asymmetric SOCs 5
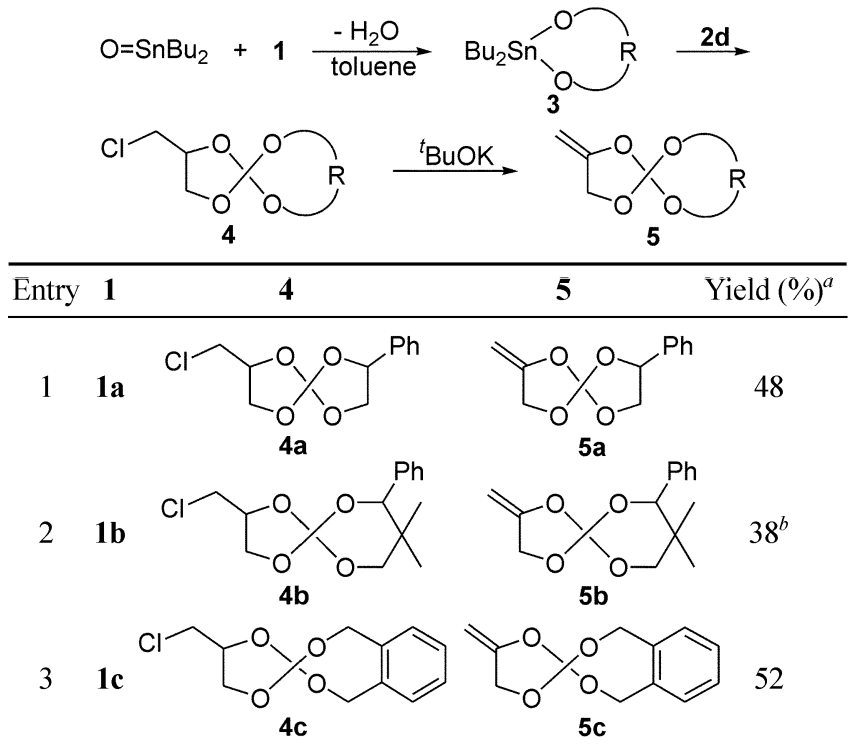

${ }^{a}$ The yields refer to isolated pure materials and based on $1 .{ }^{b} \mathrm{GC}$ Yield based on 1 .

not detected in this effort (entry 3). Thionocarbonates $\mathbf{2 a}, \mathbf{2} \mathbf{b}$. and $\mathbf{2 d}$ were easily identified since thionocarbonyl carbons are known to display weak peaks at about $190 \mathrm{ppm}$ in ${ }^{1.3} \mathrm{C}$ NMR spectra and strong peaks at $1240-1270 \mathrm{~cm}^{-1}$ in $\mathrm{F}$ ]-JR spectra.

The consecutive coupling of $\mathbf{2} \mathbf{d}$ with cyclic tin adducts $\mathbf{3}$. which are freshly made by the reaction of 1 with dibutyltin oxide, give corresponding chlorine-containing asymmetric SOCs 4. The treatment of $\mathbf{4}$ with potassium tert-butoxide in situ allows the unsaturated asymmetric SOCs $\mathbf{5}$ in good overall yields (lable 2). It is noteworthy that potassium tertbutoxide is necessary for the complete dehydrochlorination, while NaOEt give unsatisfactory results. Even though $\mathbf{5 a}$ and $5 c$ were purified by the fractional distillation and recrystallization respectively, $\mathbf{5 b}$ could not be isolated effectively. Structures of these SOCs can be easily identified by ${ }^{13} \mathrm{C}$ NMR spectra, because the spiro carbons of SOCs are appeared at $\delta 153.9 \mathrm{ppm}$ for $\mathbf{5 a}, 153.6 \mathrm{ppm}$ for $\mathbf{5 b}$, and 153.5 ppin for $\mathbf{5 c}$.

The opposite synthons were adopted to investigate the best synthetic route for the preparation of SOCs. Instead of reactions between cyclic organotins 3 and chlorothionocarbonate $2 \mathbf{d}$. couplings of chlorine-containing organotin

Table 3. Preparation of 5 via Opposite Approaches

\begin{tabular}{|c|c|c|c|}
\hline & \multicolumn{2}{|c|}{ 1) $O=S_{n n} u_{2} /$ tolune } & \\
\hline & 2) & & \\
\hline Iintry & 2 & 5 & Yield $(\%)^{t}$ \\
\hline 1 & $2 a$ & 5 a & 43 \\
\hline 2 & $2 b$ & $\mathbf{5 b}$ & $27^{6}$ \\
\hline
\end{tabular}

"The yields refer to isolated pure materials and based on 1. "GC. Yield based on 1 . with a couple of cyclic thionocarbonates 2 were performed (Table 3). Orthocarbonate $\mathbf{5 a}$ was produced successfully. while the isolation of $\mathbf{5 b}$ failed again. The yields in these opposite approaches are slightly lower than the cases of Table 2.

In conclusion, novel asymmetric SOCs bearing exomethylene groups at a to the oxygen are produced by the reaction of cyclic thionocarbonates, which are prepared by the reaction of appropriate diols with thiophosgene in the presence of DMAP, with cyclic tin compounds.

\section{Experimental Section}

Al] reactions were carried out under an inert atmosphere of $\mathrm{N}_{2}$. Toluene was distilled from sodium-benzophenone ketyl, ${ }^{1} \mathrm{H}$ NMR (300 MHz) and ${ }^{1.3} \mathrm{C}$ NMR (75 MHz) were registered in $\mathrm{CDCl}_{s}$ as solvent and tetramethylsilane (TMS) as internal standard. Analytical thin-layer chromatography (TI.C) was performed using Merck Kieselgel $60 \mathrm{~F}_{254}$ precoated plates $(0.25 \mathrm{~mm})$ with a fluorescent indicator and visualized with UV light (254 and $365 \mathrm{~nm}$ ) or by iodine vapor staining. GC analysis was performed on a bonded $5 \%$ phenylpolysiloxane BPX 5 capillary column (SGF, $30 \mathrm{~m}$, $0.32 \mathrm{~mm}$ i.d.). F:lectron impact ( $[1,70 \mathrm{eV}$ ) was used as the ionization method for the mass spectrometry. Melting points were obtained using a Barnstead/ / 'hermolyne MEL-TEMP apparatus and are uncorrected.

General Procedure for the Preparation of Cyclic Thionocarbonates 2. To the solution of diol 1 (52.2 mmol) and DMAl' (11.69 $\mathrm{g}, 95.7 \mathrm{mmol})$ in toluene $(200 \mathrm{~mL})$ was added thiophosgene $(5.00 \mathrm{~g}, 43.5 \mathrm{mmol})$ in toluene $(40.0$ $\mathrm{mL}$ ) dropwise over $15 \mathrm{~min}$. After stirred for $3 \mathrm{~h}$ at room temperature, the reaction mixture containing lots of yellow salts was diluted with toluene $(100 \mathrm{~mL})$ and transferred through a sintered glass funnel containing a small pad of silica gel. The resulting organic solution was washed with $0.1 \% \mathrm{HCl}$ aqueous solution $(60 \mathrm{~mL})$, water $(3 \times 180 \mathrm{~mL})$ and brine $(60 \mathrm{~mL})$; dried over $\mathrm{MgSO}_{4}$; and concentrated in vacuo. The crude product 2 was purified by recrystallization.

4-Phenyl-1,3-dioxa-2-cyclopentanethione (2a) was purified by recrystallization ( $\mathrm{Et}_{2} \mathrm{O}: n$-hexane $=9: 1$ ) to give a white solid $\left(7.41 \mathrm{~g}, 86 \%\right.$ ): m.p. $35-36^{\circ} \mathrm{C}$ (uncorrected); TLC $R_{i} 0.16$ (EtOAc : $n$-hexane $\left.=1: 1\right)$; ' $\mathrm{H}$ NMR $(300 \mathrm{MHz}$. CDCl $) \delta 4.56(\mathrm{dd}, J=8.81,8.17 \mathrm{~Hz}, 1 \mathrm{H}), 4.99$ (dd, $J=$ $8.82,8.48 \mathrm{~Hz}, 1 \mathrm{H}), 5.89(\mathrm{t}, J=8.23 \mathrm{~Hz}, \mathrm{l} \mathrm{H}), 7.35-7.48(\mathrm{~m}$. $5 \mathrm{H}) ;{ }^{1} \mathrm{C}$ NMR $(75 \mathrm{MHz}, \mathrm{CDCl}) \delta 75.1,83.3,126.5,129.6$, 130.3, 134.8, 191.9; F"[-IR (KBr) 1150, 1270, I380, 1450. 1710, 2360, 2980, 3030; HRMS (EI, $70 \mathrm{eV}$ ) calcd for $\mathrm{C}_{8} \mathrm{H}_{8} \mathrm{O}_{2} \mathrm{~S}\left(\mathrm{M}^{+}\right) 180.0245$, found 180.0312 .

5,5-1)imethyl--4-phenyl-1,3-dioxa-2-cyclohexanethione (2b) was purified by recrystallization $\left(\mathrm{Et}_{2} \mathrm{O}\right.$ ) to give a white crystalline solid (7.76 g. 73\%): m.p. $119-120^{\circ} \mathrm{C}$ (uncorrected); ILC $R_{f} \cdot 0.24$ (EtOAc: $n$-hexane $\left.=1: 1\right)$; 'H NMR $(300$ $\mathrm{MHz}, \mathrm{CDCl}) \delta 0.96(\mathrm{~s}, 3 \mathrm{H}), 1.04(\mathrm{~s}, 3 \mathrm{H}), 4.17(\mathrm{~d}, J=11.1$ $\mathrm{Hz}, 1 \mathrm{H}), 4.28(\mathrm{~d}, J=10.8 \mathrm{~Hz}, 1 \mathrm{H}), 5.29(\mathrm{~s}, 1 \mathrm{H}), 7.26-7.42$ $(\mathrm{m}, 5 \mathrm{H}) ;{ }^{1.7} \mathrm{C}$ NMR $\left(75 \mathrm{MHz}_{1} \mathrm{CDCl}_{3}\right) \delta 18.1,22.0,32.5$, $78.6,90.0,127.4,128.5,129.3,133.9,189.8 ;$ Fl-lR (KBr) 
$1200,1240,1290,1370,1450,2350,2880,2960,3420$. HRMS (EI, $70 \mathrm{eV}$ ) calcd for $\mathrm{C}_{12} \mathrm{H}_{14} \mathrm{O}_{2} \mathrm{~S}\left(\mathrm{M}^{-}\right) 222.0715$. found 222.0698 .

4-Chloromethyl-1,3-dioxa-2-cyclopentanethione (2d) was purified by recrystallization $\left(\mathrm{Et}_{2} \mathrm{O}: n\right.$-hexane $\left.=9: 1\right)$ to give a white crystalline solid $(6.13$ g. $84 \%)$ : m.p. $43-44^{\circ} \mathrm{C}$ (uncorrected); TLC $R_{f} 0.21$ (EtOAc : $n$-hexane $=1: 1$ ); ${ }^{1} \mathrm{H}$ NMR $\left(300 \mathrm{MHz}_{2} \mathrm{CDCl}_{3}\right) \delta 3.79-3.81(\mathrm{~m}, 2 \mathrm{H}) .4 .63+$ (dd. $J$ $=6.22 .9 .2+\mathrm{Hz} .1 \mathrm{H}), 4.783$ (t. $J=8.65 \mathrm{~Hz}, 1 \mathrm{H}) .5 .155-5.182$ (m, 1H): ${ }^{13} \mathrm{C}$ NMR $\left(75 \mathrm{MHz} . \mathrm{CDCl}_{3}\right) \delta+2.9,71.5$. 79.4 . 191.1; FT-IR (KBr) 1150. 1270. 1450, 1710. 2360, 2990. $3030 ;$ LRMS $m z$ (rel abundance) $152\left(\mathrm{M}^{+} .100\right), 103(7) .77$ (17), $75(51), 63(14) .61(17), 57(43)$.

General Procedure for the Preparation of Unsaturated Asymmetric SOCs 5. To diol 1 (31.6 mmol) in toluene (132 $\mathrm{mL})$ was added dibutyltin oxide $(7.87 \mathrm{~g}, 31.6 \mathrm{mmol})$. The resulting solution was heated at reflux for $3 \mathrm{~h}$ until the theoretical amounts of water $(0.57 \mathrm{~mL} .31 .6 \mathrm{mmol})$ was collected in the attached Dean-Stark trap. To the reaction mixture was added $2 \mathrm{~d}(4.00 \mathrm{~g}, 26.3 \mathrm{mmol})$ at room temperature. After stirring for $20 \mathrm{~h}$ at room temperature or gently heating for $t h$ depending on the solubility of 3 . the resulting mixture was treated with ${ }^{\mathrm{t}} \mathrm{BuOK}(6.50 \mathrm{~g} .57 .9$ mmol) and stirred for 2 days at rt. The reaction mixture was diluted with toluene $(100 \mathrm{~mL})$ and transferred through a sintered glass funnel containing a small pad of silica gel in order to remove salts. The resulting organic solution was washed with water $(3 \times 80 \mathrm{~mL})$ and brine $(20 \mathrm{~mL})$ : dried over $\mathrm{MgSO}_{4}$; and concentrated in vacuo. The crude product 5 was purified by either vacuum distillation or recrystallization.

2-Methylene-7-pheny]-1,4,6,9-tetraoxaspimo[4.4]nonane (5a) was purified by vacuum distillation to give a colorless oil $\left(2.60\right.$ g. $48 \%$ ): TLC $R_{f} 0.50$ (EtOAc $: n$-hexane $\left.=1: 4\right)$ : ${ }^{1} \mathrm{H}$ NMR (300 MHz. $\mathrm{CDCl}_{3}$ ) $83.96-4.04$ (m. 2H). 4.45-4.53 (m. $2 \mathrm{H}), 4.68-4.73(\mathrm{dd}, J=4.03 \mathrm{~Hz}, 2 \mathrm{H}), 4.26-4.30$ (dd. $J=$ $7.89 \mathrm{~Hz} .1 \mathrm{H}), 5.29$ (s. 1H), 7.26-7.42 (m, 5H): ${ }^{13} \mathrm{C}$ NMR $(75$ $\left.\mathrm{MHz} . \mathrm{CDCl}_{3}\right) \delta 67.2(1 \mathrm{C}), 72.2(1 \mathrm{C}), 79.1(\mathrm{lC}) .80 .7(\mathrm{lC})$. 126.5 (1C), $129.0(2 \mathrm{C}), 129.1$ (2C). $135.6(1 \mathrm{C}), 137.0$ (1C). 153.9 (1C); HRMS (EI. $70 \mathrm{eV}$ ) calcd for $\mathrm{C}_{11} \mathrm{H}_{13} \mathrm{O}_{4}\left(\mathrm{M}^{+}\right)$ 208.0736 found 208.0698 .

Spiro[2,4]-benzodioxepine-4'-methylene-3,2'-[1.3]dioxolane $(5 \mathrm{c})$ was purified by recrystallization ( $n$-hexane) to give a white solid ( 2.81 g. $52 \%$ ): m.p. $137-138^{\circ} \mathrm{C}$ (uncorrected): TLC $R_{f} 0.53$ (EtOAC: $n$-hexane $\left.=1: 4\right)$ : ${ }^{l} \mathrm{H}$ NMR $(300$ $\left.\mathrm{MHz}_{2} \mathrm{CDCl}_{\hat{j}}\right) \delta 4.04(\mathrm{~m}, \mathrm{lH})+4.50(\mathrm{~m}, \mathrm{lH}), 4.7 \mathrm{l}(\mathrm{t} . J=1.93$ Hz. 2H). 5.05 (s. 4H) $7.06-7.10$ (dd. $J=5.5+$ Hz. $2 \mathrm{H}$ ). 7.20 $7.24(\mathrm{dd} . J=5.71,2 \mathrm{H}):{ }^{13} \mathrm{C} \mathrm{NMR}\left(75 \mathrm{MHz}, \mathrm{CDCl}_{3}\right) \delta 67.17$. 67.23 (2C). 81.1 (1C). 126.4 (2C). 127.5 (2C). 130.1 (1C). 136.0 (2C). 153.6 (IC): FT-IR (KBr) 1070. 1120. 1200. $1260,1370,1450,1640,2360,2920,3440$. HRMS (EI. 70 eV) calcd for $\mathrm{C}_{11} \mathrm{H}_{12} \mathrm{O}_{4}(\mathrm{M}) 208.0736$ found 208.0721 .
Acknowledgement. This research was supported by the Chung-Ang University special grant for research equipments.

\section{References}

1. (a) Moszner. N.: Salz. U. Prog Polmm. Sci. 2001. 26.535-576. (b) Sanda. F.: Endo. T. J. Polyn. Sei. Part A. Polm. Chem. 2001. 39. 265-276. (c) Kostoryz. E. L.: Tong. P. Y.: Chappelow. C. C.: Eick. J. D.: Glaros. A. G.: Yourtee, D. 14. Dental Materials 1999, 15. 363-373. (d) Eick, J. D; Robinson, S. J.: Byerley, T. J.; Chappelow. C. C. Ouintessence Int 1993. 24,632-640.

2. Bailey. W. J.: Surl. R. L. Pohm. Prept Gm. Chent. Soc. Div Polm. Chent.) 1972. 13.281

3. (a) Endo. T.: Bailey. W. T. J. Polym. Sci. Polmm Lett. Ed. 1980. 18. 25-27. (b) Endo. T.: Okawara, M.: Yamazaki. N.; Bailey. W. J. $d$. Polym. Sci. Polym Chem Ed. 1981. 19. 1283-1286.

4. Tanigaki. K.: Saigo. K.: Ohnishi, Y.: Kato. H.: Mizutani, K. Ogasawara. T.: Endo. T. J. Appl. Polnt. Sci. 1985. 30. 1419-1428.

5. (a) Holder. A. I.: White. D. A.: Harris. C. D.: Eick. I. D.: Chappelow. C. C. J. Mole. Stuctwe (Theochem. 2001. 541. 159169. (b) Bailey. W. J. LS Patmit 4,387,215. 1983.

6. Schaefer. R; Heindl, D.: Schöde, D.: Nuyken. O.; Böhner, R:; Erdmann. C. LS Potent 5.750.590. 1998.

7. Fukuchi. I.: Sanda. F.: Endo. T. Macronolecules 2001. 34. 42964298.

8. (a) Chappelow. C. C.: Pinzino. C. S.: Teang. L.: Harris. C. D.: Holder. A. J.; Eick, J. D. J. Appl. Polym. Sci. 2000, 76. 1715-1724. (b) Wang. C. S. J. Mater Sci. Lett. 1999. 18. 1259-1262. (c) Sanda. F.; Takata. T.; Endo. T. J. Polym Sci. Part A: Polym. Chent 1994. 32. 323-332. (d) Park. Y. J.: No. K. H.: Kim. I. H.: Suh. I. H. Bull. Korean Chent. Soc. 1992. 13. 375-381. (e) No. K H. Bull. Korem Chent. Soc. 1988. 9. 183-184.

9. (a) Zhou, Z; Jin. B.: He, P. J. Appl. Polym Sci. 2002, 84, 1457 1464. (b) Komatsu. S.; Takata, T: Endo. T. Macromolecules 1993. 26, 875-878. (c) Endo. T.; Maruoka, S.: Yokozawa. T. Macronolecules 1987. 20. 2690-2693.

10. (a) Sanda. F.: Takata. T.: Endo. T. Macromol. Theory Simml. 1995. t. 221-231. (b) Moszner. N.: Zeuner. F: Rheinberger. V 1 facromol. Rapid Commm. 1995. 16. 667-672. (c) Sanda. F.: Takata. T.: Endo. T. Macromolecules 1993. 26.737-743.

11. Stansbury. J. W. J. Dent. Res. 1992. 71, 1408-1412.

12. Millich, F.: Eick. J. D.; Jeang, L.; Byerley. T. J. J. Pohm Sci. Port A. Pohm. Chem. 1993. 31. 1667-1673.

13. (a) Bromley. M. K.: Gason. S. T.: Looney. M. G.: Solomon. D. H Atst. J. Chem. 1999. 52. 657-661. (b) Bromley. M. K.: Solomon. D. H Polym 1999, 40, 6539-6543. (c) Takata. T.: Kanamaru. M: Endo. T. Macromolecules 1997, 30,6721-6726.

14. (a) Endo. T.: Bailey: W. J. J. Polnt. Sci. Pohnt Chem. Ed. 1975. 13. 2525-2530. (b) Sakai. S.: Kobayashi. Y.: Ishii. Y. J. Org. Chent 1971. 36. $1176-1180$. (c) Sakai. S.: Kiyohara. Y.: Itoh. K. Ishii. Y. J. Org. Chem. 1970. 35. 2347-2350.

15. Sakai. S; Kuroda. Y; Istii. Y. J. Org Chem 1972.37.4198-4200.

16. Stansbury. J. W. PhD. Dissertation. The University of Maryland. 1988.

17. (a) Tagishi. H.: Endo. T. J. Polm. Sci. Polm Chent Ed 1989. 27. 1415-1418. (b) Takata. T.: Endo. T. Macronolecules 1988. 21. 900-904. (c) Endo. T.: Maruoka. S.: Yokozawa. T. J. Polvm. Sci. Part A: Polym. Chem. 1987. 25. 2925-2929. (d) Endo. T: Okawara, M. Synthesis 1984. 837.

18. Bolln. C.: Frey. H.: Mülhaupt. 1facronolecules 1996. 29. 3111 3116. 